\title{
Possible hypoglycemic action of SX-fraction targeting insulin signal transduction pathway
}

This article was published in the following Dove Press journal:

International Journal of General Medicine

23 March 2013

Number of times this article has been viewed

\section{Sensuke Konno \\ Bobby Alexander \\ John Zade \\ Muhammad Choudhury \\ Department of Urology, New York Medical College, Valhalla, NY, USA}

Correspondence: Sensuke Konno Department of Urology, New York Medical College, Munger Pavilion 4th Floor, Valhalla, NY 10595, USA

$\mathrm{Tel}+$ I 9145943745

$\mathrm{Fax}+19145944428$

Email sensuke_konno@nymc.edu
Background: SX-fraction (SXF) is a bioactive glycoprotein with hypoglycemic activity that has been demonstrated in our pilot clinical study. However, how it would actually work in diabetic patients remains unclear. To explore such a mechanism, the effects of SXF on the insulin signal transduction pathway were investigated using skeletal muscle L6 cells in vitro.

Methods: L6 cells were first differentiated to myotubes expressing several biochemical parameters that were examined in this study. Myotubes were exposed to a high concentration ( $35 \mathrm{mM})$ of glucose (Glc) alone or in combination with SXF or insulin for 24 hours. Possible effects of these agents on activities of insulin receptor (IR), IR substrate 1 (IRS-1), and Akt, which are key elements involved in the signal pathway, were assessed using enzyme-linked immunosorbent assay (ELISA). Any changes in Glc uptake were also determined.

Results: High Glc indeed led to inactivation of IR, IRS-1, and subsequent Akt in myotubes, indicating an interruption of the signal pathway. However, such inactivation was reversed or reactivated by SXF, presumably aiding the occurrence of successive signaling events. Measurement of Glc uptake to assess the outcome of this signaling cascade showed that high Glc decreased Glc uptake (interfering with the signal pathway), but SXF was capable of overcoming such a suppressive effect, resulting in the increased Glc uptake. Insulin was used as a positive control in this study and all results were nearly compatible to those obtained from SXF.

Conclusion: The present study suggests that SXF may specifically target the insulin signal pathway, and, in particular, the IR and IRS-1 therein that trigger the subsequent signaling events. As a result, SXF could activate such an impaired signal pathway through high Glc or under a hyperglycemic milieu, thereby ultimately facilitating Glc uptake. This may then account for possible hypoglycemic action of SXF.

Keywords: diabetes, insulin resistance, mushroom extract, natural remedy, mode of action

\section{Introduction}

Diabetes mellitus is currently classified into two types: type 1 (insulin-dependent diabetes) and type 2 (non-insulin-dependent diabetes). ${ }^{1}$ Type 1 diabetes is primarily linked to insulin deficiency (due to dysfunction of pancreatic $\beta$-cells), representing $<20 \%$ of all diabetic cases. ${ }^{2}$ In contrast, type 2 diabetes with a $>80 \%$ incidence rate involves multiple factors, such as defects in insulin secretion, insulin resistance at peripheral sites, and elevated hepatic glucose (Glc) production. ${ }^{2,3}$ Hence, type 2 diabetes has a higher prevalence with more complex etiologies compared to type 1 . In addition, type 1 diabetes is more manageable through insulin injection, whereas the primary problem with type 2 diabetes is not insulin deficiency but insulin resistance, making it more difficult to treat. $^{3}$ In fact, because of this insulin resistance, current oral therapy using sulfonylurea 
derivatives, ${ }^{4}$ which mainly stimulate insulin secretion from pancreatic $\beta$-cells, often fails to achieve the expected level of efficacy. To enhance peripheral insulin sensitivity, pharmaceuticals such as troglitazone ${ }^{5}$ and metformin ${ }^{6}$ have also been developed. Although some improved glycemic control with these drugs has been reported, potential adverse effects had to be advised. ${ }^{7}$ Thus, it has become necessary to establish alternative means or find safer and more effective agents with which to overcome insulin resistance. This could be the most rational approach for improving treatment for type 2 diabetic patients.

SX-fraction (SXF) is a water-soluble bioactive glycoprotein with a $\sim 20,000$ Da molecular weight, that has exhibited hypoglycemic activity in diabetic mice and in a few clinical studies of type 2 patients. ${ }^{8-11}$ Our clinical study shows that all seven diabetic patients demonstrated over 30\% (30\%-63\%) decline in their fasting blood Glc (FBG) levels under a SXF regimen of 2-4 weeks (Table 1). None of the participants presented palpable ailments or adverse effects linked to SXF during the trial, further demonstrating its safety in human use. However, the hypoglycemic mechanism of SXF or how it would work is not yet fully understood. We then hypothesized that SXF might enhance insulin sensitization by acting on the insulin signal transduction pathway.

Currently, how or why insulin-targeting tissues/organs would become insulin resistant remains unknown, but it has been postulated that the insulin receptor (IR) might present an important clue. ${ }^{12}$ The IR is a heterotetrameric glycoprotein consisting of two $\alpha$-subunits and two $\beta$-subunits. ${ }^{12}$ The binding of insulin to the $\alpha$-subunits of the IR induces a conformational change that leads to trans-autophosphorylation of tyrosine residues on the $\beta$-subunits, activating their tyrosine kinase activity. ${ }^{12,13}$ One such tyrosine residue, tyrosine 972 , when being phosphorylated, serves as a binding site for the

Table I Hypoglycemic effects of SXF on type 2 diabetic patients

\begin{tabular}{|c|c|c|c|c|}
\hline \multicolumn{2}{|l|}{ Patients } & \multicolumn{2}{|c|}{ Average FBG (mg/dL) } & \multirow{2}{*}{$\begin{array}{l}\text { \% FBG decline } \\
\text { with SXF }^{\mathrm{a}}\end{array}$} \\
\hline Age (years) & Sex & Before SXF & After SXF & \\
\hline 44 & M & $\sim 260$ & $90-100$ & $\sim 63$ \\
\hline 75 & $\mathrm{~F}$ & $\sim 200$ & $110-130$ & $\sim 40$ \\
\hline 25 & $\mathrm{~F}$ & $150-180$ & $110-120$ & $\sim 30$ \\
\hline 37 & M & $180-200$ & $|20-| 40$ & $\sim 32$ \\
\hline 41 & M & $\sim 210$ & $100-110$ & $\sim 50$ \\
\hline 64 & $\mathrm{~F}$ & $\sim 220$ & $130-150$ & $\sim 37$ \\
\hline 53 & $\mathrm{~F}$ & $170-190$ & $100-110$ & $\sim 42$ \\
\hline
\end{tabular}

Note: ${ }^{a}$ As all patients were under oral medication therapy, their average FBG levels before SXF were with medications while those after SXF were with both medications and SXF. Hence, the percent of FBG decline with SXF reflects the improved glycemic control with SXF.

Abbreviations: $F$, female; FBG, fasting blood glucose; M, male; SXF, SX-fraction. phosphotyrosine binding domains of IR substrate 1 (IRS1), whose tyrosine residues are then phosphorylated. ${ }^{12,14}$ This tyrosine-phosphorylated IRS-1 acts as a docking site/molecule that binds to and activates phosphatidylinositol 3-kinase (PI3K), which in turn activates serine/threonine kinase Akt (protein kinase B). ${ }^{12,13,15,16}$ Activated Akt induces the translocation of Glc transporter 4 (GLUT4) to the plasma membrane, ultimately promoting Glc uptake by insulin responsive cells..$^{13,15,17}$ This is rather a simplified scheme of the insulin signal transduction pathway, which is triggered by activation of the IR (with insulin) and undergoes the cascade of biochemical events described above. Two other insulin signal pathways have also been postulated; ${ }^{14,15}$ it is indeed a complex biochemical process and more studies are required for further elaboration. Nevertheless, insulin resistance (of the IR) developed by the undefined (acquired and inherited) causes would block the signal pathway, resulting in an accumulation of Glc in the circulation (ie, hyperglycemia). It is then possible that a prolonged hyperglycemic milieu may further inactivate or keep insensitizing the IR/ IRS-1, thereby developing into the chronic hyperglycemic condition (ie, diabetes). Thus, one rational approach to overcoming such insulin resistance would be by (re)activating the IR/IRS-1 to successfully execute the entire signal transduction pathway.

Accordingly, we investigated the effects of Glc on the insulin signal pathway and if hypoglycemic action of SXF might also target the same pathway. Particularly, we focused on the effects of Glc and/or SXF on the phosphorylation status of IR, IRS-1, and Akt, which would specially indicate their activation status. The skeletal muscle L6 cells were used as an in vitro model in this study. ${ }^{18}$ In addition, a Glc uptake study ${ }^{17,18}$ was performed to assess the potential improvement in Glc uptake with SXF. More details and notable findings are described and discussed herein.

\section{Materials and methods Cell culture}

Rat skeletal muscle L6 cells were obtained from the American Type Culture Collection ([ATCC] Manassas, VA, USA) and maintained in RPMI 1640 medium containing 10\% fetal bovine serum (FBS), penicillin (100 U/mL), and streptomy$\operatorname{cin}(100 \mu \mathrm{g} / \mathrm{mL})$. SXF was a kind gift from the manufacturer (Mushroom Wisdom, Inc, East Rutherford, NJ, USA). For experiments, each $5 \mathrm{~mL}$ cell culture was placed in T-25 culture flasks at the initial cell density of $5 \times 10^{4}$ cells/ $\mathrm{mL}$ until they reached $\sim 90 \%$ confluence (usually within 3 days). Spent medium was discarded and fresh Dulbecco's 
modified Eagle's medium (DMEM) containing 2\% FBS (with penicillin and streptomycin) was added to all flasks and incubated until they were differentiated to myotubes expressing IR, IRS-1, and other biochemical parameters. ${ }^{18}$ This differentiation process usually took 7-10 days, and DMEM was replenished every 3-4 days. Once L6 cells were differentiated (after $\sim 2$ weeks), they were first treated with Glc for 24 hours and then treated with SXF or insulin for 15 minutes. Following this brief incubation, all cells were harvested and stored at $-80^{\circ} \mathrm{C}$ until use.

\section{Preparation of cell lysates (cytosolic proteins)}

Cell lysis was performed on ice, following the manufacturer's protocol (Life Technologies, Carlsbad, CA, USA). Harvested cells (cell pellets) from control or agent-treated cells were resuspended in cell extraction buffer and lysed by 30-minute incubation on ice with vortexing every 10 minutes. Cell lysates were then obtained by centrifugation at $13,000 \mathrm{rpm}$ for 10 minutes at $4^{\circ} \mathrm{C}$, and the protein concentration of each sample was determined using Pierce Protein Assay Reagent (Thermo Scientific, Rockford, IL, USA) on a spectrophotometer. All cell lysates were stored at $-80^{\circ} \mathrm{C}$ until use.

\section{Enzyme-linked immunosorbent assay (ELISA)}

This assay was performed following the manufacturer's protocol (Life Technologies). Briefly, $40 \mu \mathrm{g}$ of cell lysates prepared above was added to the 96-well plate coated with specific antibodies, ie, anti-IR tyrosine residue (y), -IRS-1 serine residue (s), or -Akt serine residue (s), and incubated for 2 hours at room temperature (RT) to allow the antigen-antibody binding. Several different concentrations of standards for each of IR(y), IRS-1(s), and Akt(s) were also run in parallel. After discarding cell lysates and washing sample wells, antibody detection solution was added to the plate, followed by 1-hour incubation at RT. After removing solution and washing wells, antibody conjugate (anti-rabbit IgG-HRP) was added to the plate that was incubated for 30 minutes at RT. After washing wells, chromogen solution was added to all wells, which were then incubated in the dark for 30 minutes at RT to yield blue-colored products for detection. Following 30-minute incubation, stop solution was added to all wells (changed to yellow color) to terminate the colorimetric reaction. The intensities of colored products were then quantitated using a microplate reader at $450 \mathrm{~nm}$. The phosphorylation levels of IR(y), IRS-1(s), or Akt(s) were estimated relative to those of the respective standards.

\section{Measurement of 2-deoxyglucose (DOG) uptake}

Assay for 2-DOG uptake was performed following the method of Prabhakar and Doble, ${ }^{19}$ with some modifications. Differentiated cells in a 6-well plate were treated with high Glc for 24 hours. All wells were then washed thoroughly with Krebs-Ringer-Phosphate (KRP) buffer (20 mM HEPES, pH 7.4, $120 \mathrm{mM} \mathrm{NaCl}, 5 \mathrm{mM} \mathrm{KCl}, 2 \mathrm{mM} \mathrm{CaCl}_{2}, 1.5 \mathrm{mM} \mathrm{MgSO}_{4}$, $1.5 \mathrm{mM} \mathrm{KH}_{2} \mathrm{PO}_{4}$ ) to remove residual Glc. One milliliter of KRP buffer containing $0.1 \%$ bovine serum albumin (BSA) $(\mathrm{w} / \mathrm{v})$ was added to each well and the cells in specified wells were also exposed to SXF or insulin for 15 minutes. After this brief exposure, Glc uptake was initiated by adding a radioactive ligand, 2-deoxy-D-[1- $\left.{ }^{3} \mathrm{H}\right]$-glucose $\left(\left[{ }^{3} \mathrm{H}\right]-\mathrm{DOG}\right.$; specific activity of $10 \mathrm{Ci} / \mathrm{mmol}$ ), to all wells and incubated for 20 minutes at $37^{\circ} \mathrm{C}$. Cells were washed three times with ice-cold phosphatebuffered saline (PBS) and solubilized in $0.1 \mathrm{~N} \mathrm{NaOH}$. A $200 \mu \mathrm{L}$ aliquot of cell lysis was measured, using a scintillation counter, for the radioactivity ([$\left.\left.{ }^{3} \mathrm{H}\right]-\mathrm{DOG}\right)$ incorporated into (taken up by) the cells. Background radioactive count (cpm) was determined by a blank without cell lysis and all counts were normalized by subtracting this baseline cpm. The amount of Glc uptake was then expressed by the percent relative to controls (100\%).

\section{Statistical analysis}

All data were presented as mean \pm standard deviation (SD), and statistical differences between groups were assessed with either one-way analysis of variance (ANOVA) or the unpaired Student's $t$-test. Values of $P<0.05$ were considered to indicate statistical significance.

\section{Results Effects of Glc on IR(y) and IRS-I (s) in differentiated $\mathrm{L} 6$ cells (myotubes)}

We first examined whether antibodies we would use in this study were indeed specific and appropriate for detecting the IR(y) and IRS-1(s) in differentiated L6 cells (myotubes). These were exposed to three different concentrations of Glclow $(5.5 \mathrm{mM})$, medium $(20 \mathrm{mM})$, or high $(35 \mathrm{mM})$ - and the phosphorylation status of IR(y) and IRS-1(s), which would directly reflect their biological activities, was analyzed at 24 hours using ELISA.

Figure 1 shows that the phosphorylation level of IR(y) declined, while that of IRS-1(s) elevated as Glc 


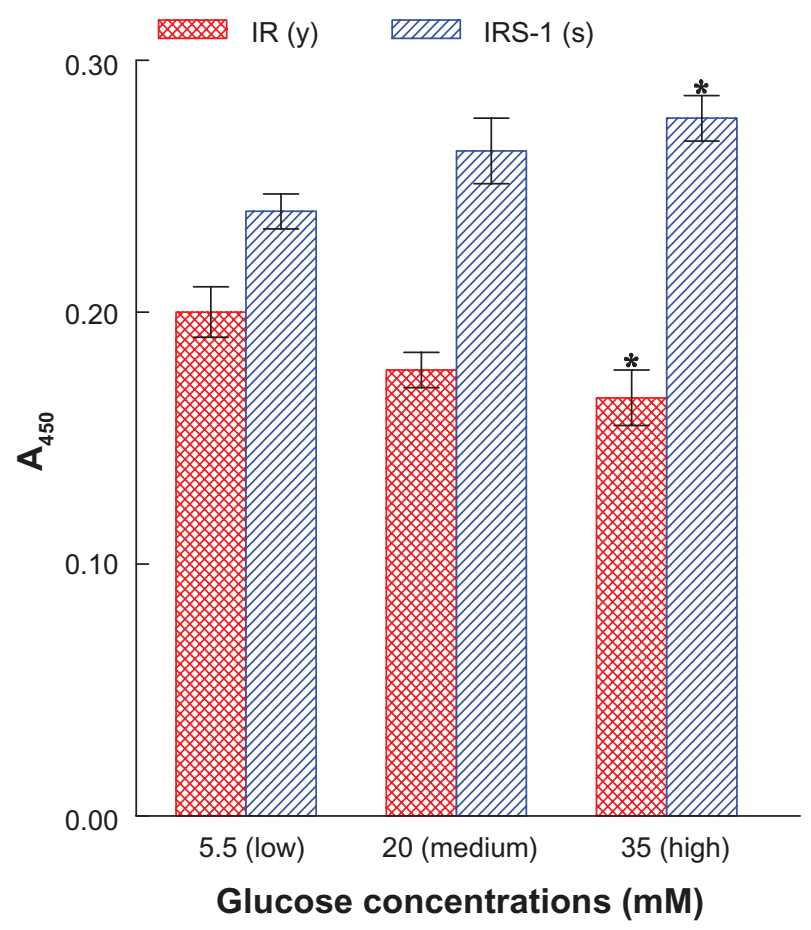

Figure I Effects of varying concentrations of Glc on the phosphorylation levels of IR(y) or IRS-I(s) in L6 myotubes.

Notes: After cells were exposed to low (as control, $5.5 \mathrm{mM})$, medium $(20 \mathrm{mM})$, or high ( $35 \mathrm{mM}$ ) concentrations of Glc for 24 hours, the phosphorylation status of $I R(y)$ or IRS-I(s) was analyzed and expressed by the absorbance readings at $450 \mathrm{~nm}$. All data are mean \pm SD from three separate experiments ( $* P<0.05$ versus respective control).

Abbreviations: $A_{450}$, absorbance at $450 \mathrm{~nm}$; Glc, glucose; IR, insulin receptor; IRS- I, IR substrate I; (s), serine residue; SD, standard deviation; (y), tyrosine residue.

concentrations increased. Such a decrease in $\operatorname{IR}(y)$ and increase in IRS-1(s) phosphorylation would indicate inactivation of IR and IRS-1, ${ }^{12,16}$ which could then prevent the subsequent signaling events. For the signal pathway to proceed, IR(y) must be preferentially more phosphorylated and activated while IRS-1(s) must be less phosphorylated and inactivated. Meanwhile, as high (35 mM) Glc had the most distinctive effects, the rest of our study was performed with this Glc concentration.

\section{Effects of SXF or insulin on IR(y) phosphorylation under high Glc}

We examined the effects of SXF or insulin (as a positive control) on the phosphorylation status of IR(y) in the presence of high Glc (35 mM) in differentiated cells (myotubes). Following 24-hour incubation, the cells treated with Glc were exposed to SXF $(300 \mu \mathrm{g} / \mathrm{mL})$ or insulin $(100 \mathrm{nM})$ for 15 minutes. After a brief 15-minute exposure, all cells were harvested and their cell lysates were subjected to ELISA for IR(y). It should be noted that our pilot study confirmed that 15-minute exposure was as good/effective as 30 or
60 minutes with no apparent differences in the outcomes (data not shown).

Figure 2 shows that the IR(y) phosphorylation level of control cells was decreased by $\sim 15 \%$ with 24 -hour Glc treatment $(P<0.05)$, indicating the loss of IR activity. However, SXF was able to elevate the reduced IR(y) level to $\sim 10 \%$ higher than controls or $\sim 29 \%$ greater than Glc-suppressed cell group $(P<0.05)$. This elevated IR(y) level with SXF was also as high as that with insulin exposure $(P<0.05)$. Thus, these results suggest that SXF may reactivate Glcinactivated IR capable of transducing the signal to carry on the subsequent events.

\section{Effects of SXF or insulin on IRS-I(s) phosphorylation}

We next examined the effects of SXF or insulin on IRS-1(s) phosphorylation. Cells treated with Glc for 24 hours were briefly (15 minutes) exposed to SXF $(300 \mu \mathrm{g} / \mathrm{mL})$ or insulin (100 nM), followed by ELISA for IRS-1(s).

Figure 3 shows that high Glc increased IRS-1(s) phosphorylation by $\sim 12 \%$ higher than controls $(P<0.05)$, indicating inactivation of IRS-1. Yet, SXF was capable of reducing such an elevated IRS-1(s) level by $\sim 11 \%$ lower

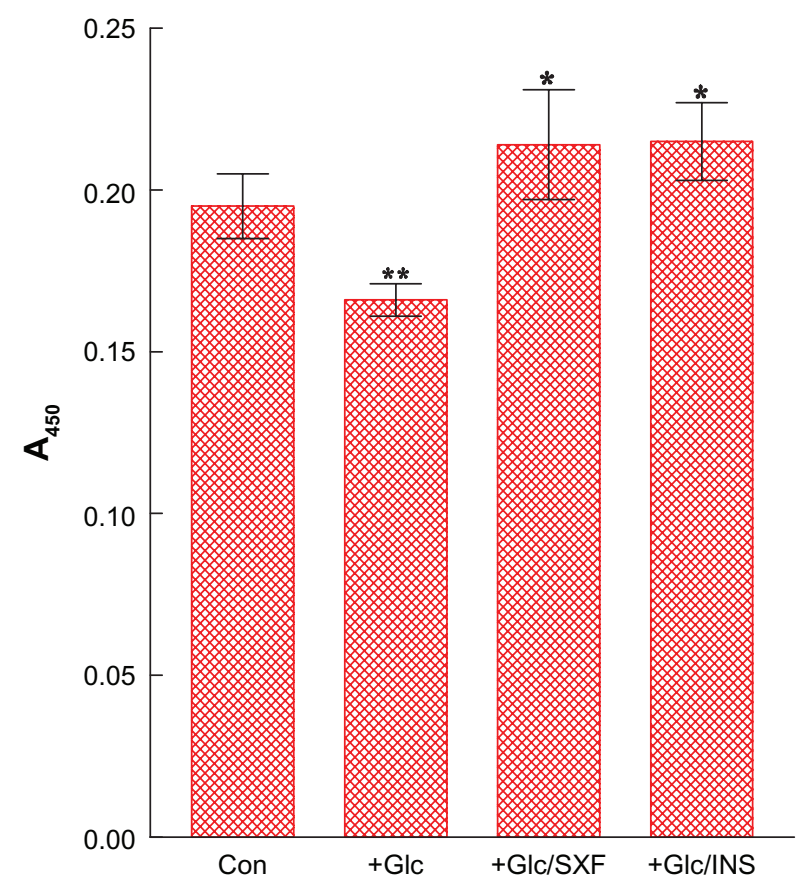

Figure 2 Effects of SXF or INS on IR(y) phosphorylation under high (35 mM) Glc. Notes: Following 24-hour high Glc treatment, cells were exposed to SXF $(300 \mu \mathrm{g} / \mathrm{mL})$ or INS (100 nM) for 15 minutes and IR(y) was analyzed using ELISA. All data are mean \pm SD from three independent experiments $(* P<0.05$ versus Glctreated or $* * P<0.05$ versus control).

Abbreviations: $A_{450}$, absorbance at $450 \mathrm{~nm}$; Con, control; ELISA, enzyme-linked immunosorbent assay; Glc, glucose; INS, insulin; IR, insulin receptor; SD, standard deviation; SXF, SX-fraction; (y), tyrosine residue. 


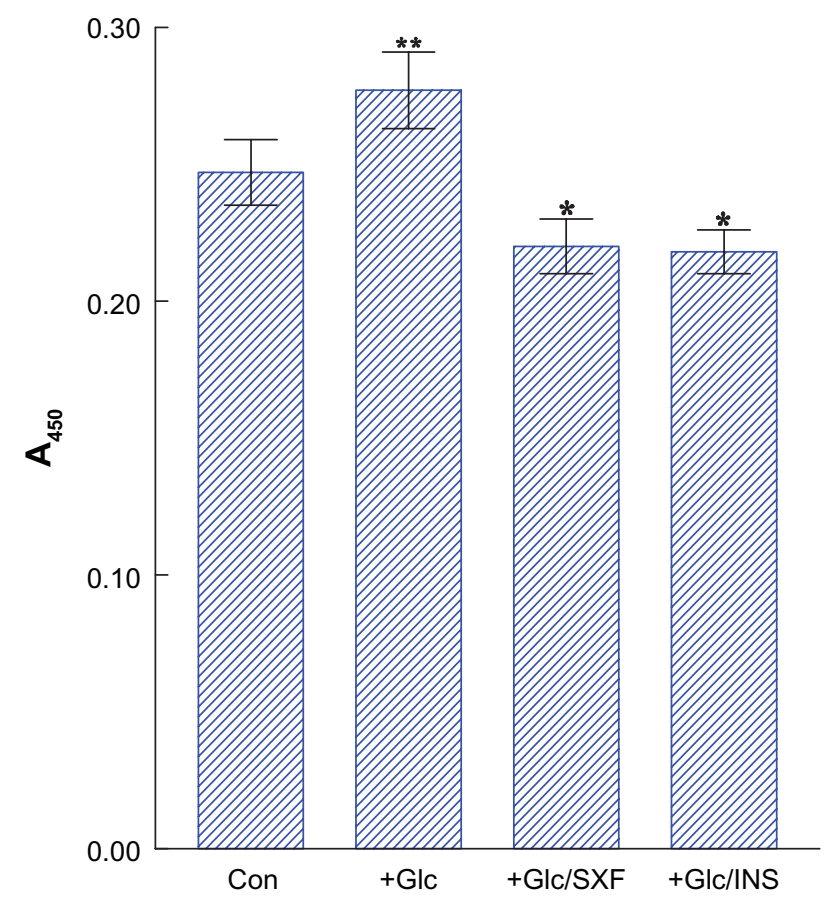

Figure 3 Effects of SXF or INS on IRS-I (s) phosphorylation under high Glc. Notes: Following 24-hour high Glc treatment, cells were exposed to SXF $(300 \mu \mathrm{g} / \mathrm{mL})$ or INS (I00 nM) for I 5 minutes and IRS-I (s) was analyzed using ELISA All data are mean \pm SD from three separate experiments $\left({ }^{*} P<0.05\right.$ versus Glctreated or **P $<0.05$ versus control).

Abbreviations: $A_{450}$, absorbance at $450 \mathrm{~nm}$; Con, control; ELISA, enzyme-linked immunosorbent assay; Glc, glucose; INS, insulin; IRS- I, insulin receptor substrate I; (s), serine residue; SD, standard deviation; SXF, SX-fraction.

than controls or $\sim 20 \%$ lower than a Glc-enhanced cell group $(P<0.05)$. Insulin also reduced the IRS-1(s) level similar to that of SXF exposure $(P<0.05)$. Since this reduction in IRS-1(s) phosphorylation with SXF indicates activation of IRS- $1,{ }^{16}$ the subsequent signaling events would presumably be carried out.

\section{Effects of SXF or insulin on Akt(s) phosphorylation}

To assess continuity of the signaling cascade, the effects of SXF or insulin were examined on the phosphorylation status of Akt(s), because Akt is one of the key intermediate components in the signal pathway and its increased phosphorylation level (mediated through PI3K) is indicative of its activation. ${ }^{13,17}$ Following 24-hour Glc treatment, those cells were briefly ( 15 minutes) exposed to SXF ( $300 \mu \mathrm{g} / \mathrm{mL})$ or insulin $(100 \mathrm{nM})$, followed by ELISA for Akt(s).

Figure 4 shows that the phosphorylation level of Akt(s) in Glc-treated cells was $\sim 42 \%$ lower than in controls $(P<0.03)$, indicating inactivation of Akt. However, this Akt inactivation (by Glc) was prevented with SXF, elevating the Glc-reduced Akt activity to $\sim 2$-fold (in Glc/SXF-treated cells) or to $\sim 18 \%$

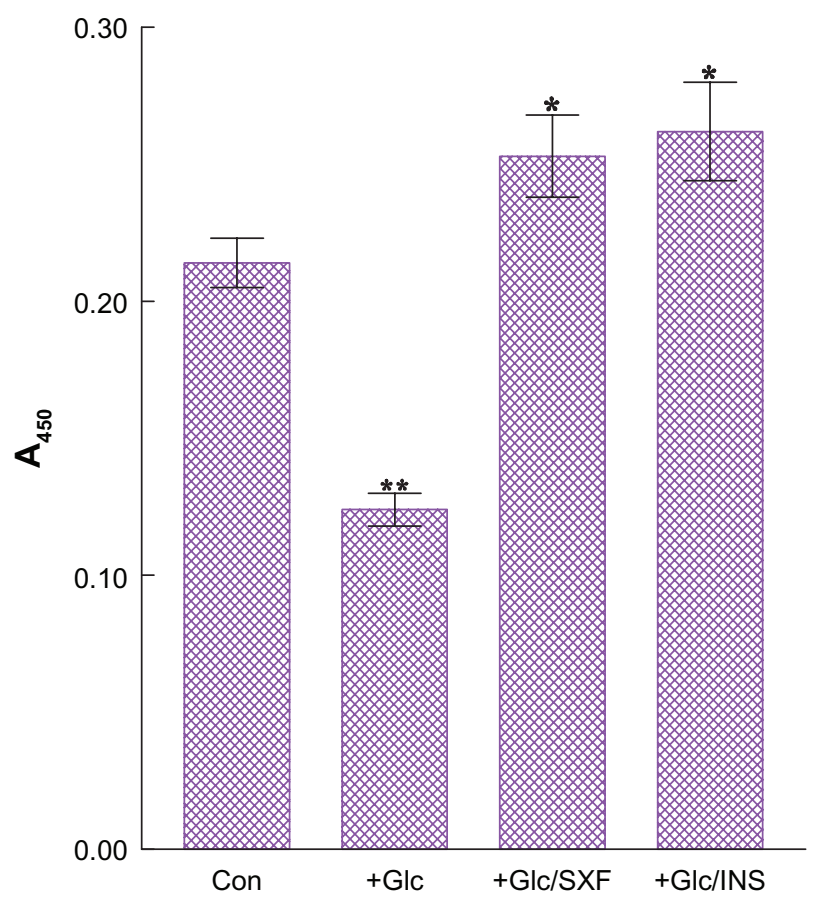

Figure 4 Effects of SXF or INS on Akt(s) phosphorylation under high Glc. Notes: Following 24-hour high Glc treatment, cells were exposed to SXF $(300 \mu \mathrm{g} / \mathrm{mL})$ or INS $(100 \mathrm{nM})$ for 15 minutes and Akt(s) was analyzed using ELISA. All data are mean \pm SD from three separate experiments $(* P<0.01$ versus Glctreated or $* * P<0.03$ versus control).

Abbreviations: $A_{450}$, absorbance at $450 \mathrm{~nm}$; Con, control; ELISA, enzyme-linked immunosorbent assay; Glc, glucose; INS, insulin; (s), serine residue; SD, standard deviation; SXF, SX-fraction.

higher than in controls $(P<0.01)$. Similarly, insulin was capable of raising the Glc-reduced Akt(s) level to $\sim 2.1$-fold (in Glc/INS-treated cells) or to $\sim 22 \%$ higher than in controls $(P<0.01)$. These results thus suggest that the signaling cascade was continuously carried out (with SXF) and would be continued further.

\section{Effects of SXF or insulin on Glc uptake}

Lastly, how SXF or insulin would affect Glc uptake under high Glc was examined to assess the outcome of the completed signaling cascade. After 24-hour Glc treatment, cells were exposed to SXF $(300 \mu \mathrm{g} / \mathrm{mL})$ or insulin $(100 \mathrm{nM})$ for 15 minutes, followed by Glc-uptake assay using $\left({ }^{3} \mathrm{H}\right)$-DOG.

Figure 5 shows that the $\sim 24 \%$ reduction in Glc uptake $(P<0.05)$ with high Glc (compared to controls) was reversed with SXF, stimulating such a reduction to the $\sim 1.5$-fold greater uptake level (in Glc/SXF-treated cells) or to $\sim 17 \%$ higher than in controls $(P<0.03)$. As expected, insulin demonstrated the best stimulatory effects, with $\sim 1.7$-fold higher Glc uptake (in Glc/INS-treated cells) than in the Glc-reduced 


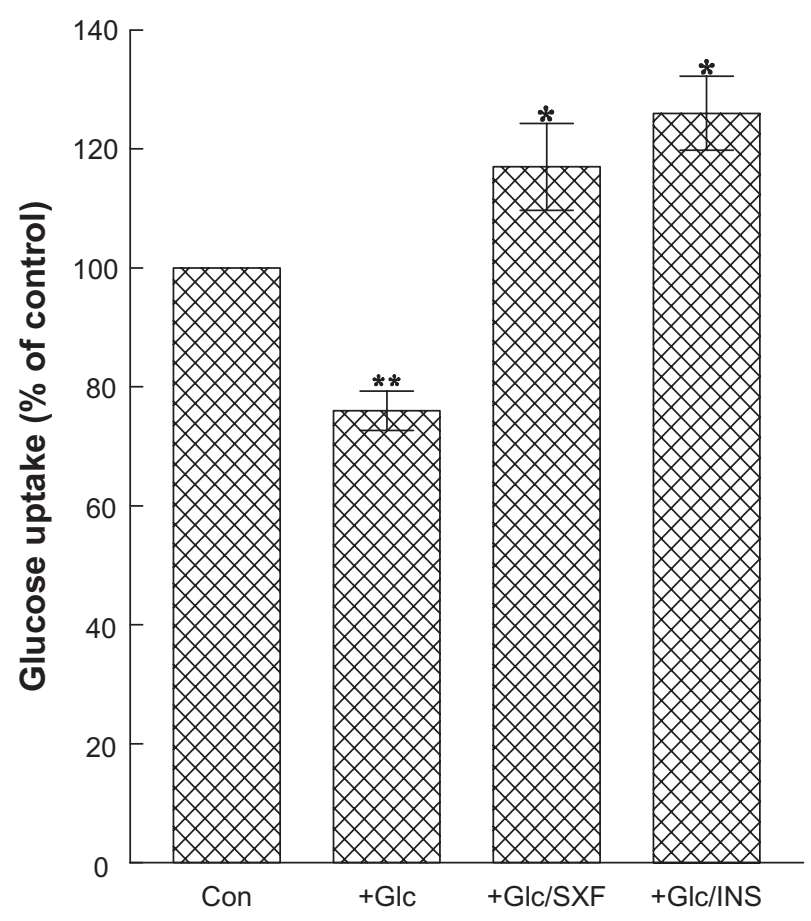

Figure 5 Effects of SXF or INS on glucose uptake.

Notes: Following 24-hour Glc treatment and 15-minute SXF or INS exposure, Glc uptake was measured using a radioactive ligand and expressed by the percent relative to control (100\%). All data are mean \pm SD from three separate experiments ( $* P<0.03$ versus Glc-treated or **P $<0.05$ versus control).

Abbreviations: Con, control; Glc, glucose; INS, insulin; SD, standard deviation; SXF, SX-fraction.

group as well as $\sim 26 \%$ higher Glc uptake than in controls $(P<0.03)$. Overall, initial activation of IR/IRS-1 with SXF may thus account for the successful completion of the signal pathway with increased Glc uptake.

\section{Discussion}

To improve the efficacy of current treatment for type 2 diabetes, the potential effects of SXF extracted from maitake mushrooms have been investigated in animal and limited clinical settings. ${ }^{9-11}$ Particularly, it was encouraging that the glycemic control of several diabetic patients under oral medications was significantly improved with a SXF regimen (Table 1). While more controlled clinical trials on SXF need to be conducted to further verify its hypoglycemic effect, few studies have yet been performed to unveil the mode of hypoglycemic action of SXF. To gain an insight into the hypoglycemic mechanism of SXF, we investigated its effects on the insulin signal transduction pathway, ie, a cascade of biochemical events required for the glycemic control in response to insulin. ${ }^{13,15}$

Insulin resistance is a common but complex pathological state and a major factor for developing type 2 diabetes, resulting in a dysfunction or impairment of the insulin signal pathway. ${ }^{13-16}$ We thus investigated if SXF might positively act on the impaired signal pathway (under a high-Glc milieu) to overcome insulin resistance using an in vitro model. Our primary objective was to assess the effects of SXF on three key components, IR, IRS-1, and Akt, which play a pivotal role in the signal pathway. Such study may then provide us with valuable information.

We first examined how a prolonged hyperglycemic condition would actually affect biological activities of IR, IRS-1, or Akt. Our study showed that high Glc (35 mM) significantly inactivated both IR and IRS-1 in 24 hours, indicated by the decrease in IR(y) and the increase in IRS-1(s) phosphorylation levels, respectively (Figures 2 and 3 ). It should be clarified that, generally, the phosphorylation status of the tyrosine residues in both IR(y) and IRS-1(y) would be assessed for their activities; however, the serine phosphorylation status was examined in IRS-1 instead, due to unavailability of appropriate anti-tyrosine antibody in this study. It has been shown that IRS-1 contained several tyrosine phosphorylation sites and 50 potential serine/ threonine phosphorylation sites, ${ }^{16}$ and serine phosphorylation of IRS-1 would impair its tyrosine phosphorylation, causing it to become inactivated and serve as a poor IR substrate. ${ }^{20}$ Such serine-phosphorylated IRS-1 may then attenuate the rest of the signal pathway (whereas tyrosine-phosphorylated IRS-1 would facilitate the pathway). It is thus plausible that a fine balance between tyrosine and serine phosphorylation of IRS-1 will regulate the IRS-1 activity and functions. Change in this equilibrium could also lead to pathological conditions such as insulin resistance. In fact, the development of insulin resistance is shown to be associated with serine/threonine phosphorylation of IRS-1; ${ }^{13,14,21}$ after all, although high Glc inactivated both IR and IRS-1, SXF was capable of (re)activating them and facilitating the signaling cascade.

While activation of IR and IRS-1 with SXF (under high Glc) was considered the early critical step in the signal pathway, it was also important to assess the status of another key element, Akt, during the progress of the signaling events. Actually, activated IRS-1 is responsible for activation of PI3K, which in turn promotes serine/threonine phosphorylation of Akt for its activation. ${ }^{13,15,17}$ This Akt activation is crucial because it would not only advance the pathway, but also facilitate/induce the final stage of the pathway, ie, the translocation of GLUT 4. ${ }^{13,17}$ Although high Glc inactivated Akt, SXF was capable of significantly (re)activating it, evidenced by the increased Akt(s) phosphorylation level (Figure 4). This finding suggests that the signal pathway continued successfully through activation of PI3K, which was essentially required for subsequent activation of Akt. 
Finally, Glc uptake was measured to evaluate the overall outcomes of the SXF-initiated signal pathway under high Glc. Although the amount of Glc uptake by the cells was considerably reduced with high Glc, SXF promoted this impaired Glc uptake greatly as its level reached even higher than in controls. Thus, these results suggest that the signal pathway being impaired with high Glc could be (re) activated or turned on by SXF and successfully completed with increased Glc uptake.

We assume that this increased Glc uptake (with SXF) would presumably result from GLUT 4 translocation to the plasma membrane, which is induced by activated Akt, ${ }^{17}$ although we did not examine the cellular localization of GLUT4 in this study. For further confirmation, such a study is currently underway in our laboratory.

\section{Conclusion}

SXF may primarily target or act on the insulin signal transduction pathway, overcoming insulin resistance (of IR and IRS-1) to carry on the subsequent signaling events. This may account for the possible hypoglycemic action of SXF.

\section{Acknowledgments}

The authors thank Mr Mike Shirota (Mushroom Wisdom, Inc) for generously providing SX-fraction for this study.

\section{Disclosure}

The authors report no conflicts of interest in this work.

\section{References}

1. American Diabetes Association (ADA). Standards of medical care in diabetes 2011. Diabetes Care. 2012;34:S11-S63.

2. Caro JF. Effects of glyburide on carbohydrate metabolism and insulin action in the liver. Am J Med. 1990;89:17S-25S.

3. Groop LC. Insulin resistance: the fundamental trigger of type 2 diabetes. Diabetes Obes Metab. 1999;1:S1-S7.

4. Groop LC. Sulfonylureas in NIDDM. Diabetes Care. 1992;15: 737-754.
5. Nolan JJ, Ludvik B, Beerdsen P, Joyce M, Olefsky J. Improvement in glucose tolerance and insulin resistance in obese subjects treated with troglitazone. N Engl J Med. 1994;331:1188-1193.

6. DeFronzo RA, Goodman AM. Efficacy of metformin in patients with non-insulin-dependent diabetes mellitus. The Multicenter Metformin Study Group. N Engl J Med. 1995;333:541-549.

7. Bailey CJ. Biguanides and NIDDM. Diabetes Care. 1992;15: 755-772.

8. Kubo K, Aoki H, Nanba H. Anti-diabetic activity present in the fruit body of Grifola frondosa (Maitake). Biol Pharm Bull. 1994;17:1106-1110.

9. Konno S, Tortorelis DG, Fullerton SA, Samadi AA, Hettiarachchi J, Tazaki H. A possible hypoglycaemic effect of maitake mushroom on type 2 diabetic patients. Diabet Med. 2001;18:1010.

10. Manohar V, Talpur NA, Echard BW, Lieberman S, Preuss HG. Effects of a water-soluble extract of maitake mushroom on circulating glucose/insulin concentrations in KK mice. Diabetes Obes Metab. 2002;4:43-48.

11. Preuss HG, Echard B, Bagchi D, Perricone NV, Zhuang C. Enhanced insulin-hypoglycemic activity in rats consuming a specific glycoprotein extracted from maitake mushroom. Mol Cell Biochem. 2007;306: 105-113.

12. Youngren JF. Regulation of insulin receptor function. Cell Mol Life Sci. 2007;64:873-891.

13. Biddinger SB, Khan CR. From mice to men: insight into insulin resistance syndromes. Annu Rev Physiol. 2006;68:123-158.

14. Boura-Halfon S, Zick Y. Phosphorylation of IRS proteins, insulin action, and insulin resistance. Am J Physiol Endocrinol Metab. 2009; 296:E581-E591.

15. Saltiel AR, Khan CR. Insulin signaling and the regulation of glucose and lipid metabolism. Nature. 2001;414:799-806.

16. Gual P, Le Marchand-Brustel Y, Tanti JF. Positive and negative regulation of insulin signaling through IRS-1 phosphorylation. Biochimie. 2005;87:99-109.

17. Kohn AD, Summers SA, Birnbaum MJ, Roth RA. Expression of a constitutively active Akt Ser/Thr kinase in 3T3-L1 adipocytes stimulates glucose uptake and glucose transporter 4 translocation. $J$ Biol Chem. 1996;271:31372-31378.

18. Mitsumoto Y, Burdett E, Grant A, Klip A. Differential expression of the GLUT1 and GLUT4 glucose transporters during differentiation of L6 muscle cells. Biochem Biophys Res Commun. 1991;175:652-659.

19. Prabhakar PK, Doble M. Synergistic effect of phytochemicals in combination with hypoglycemic drugs on glucose uptake in myotubes. Phytomedicine. 2009;16:1119-1126.

20. Tanti JF, Gremeaux T, Van Obberghen E, Le Marchand-Brustel Y. Serine/ threonine phosphorylation of insulin receptor substrate 1 modulates insulin receptor signaling. J Biol Chem. 1994;269:6051-6057.

21. Craparo A, Freund R, Gustafson TA. 14-13-3 (epsilon) interacts with the insulin-like growth factor 1 receptor and insulin receptor substrate 1 in a phosphoserine-dependent manner. J Biol Chem. 1997;272: 11663-11669.
International Journal of General Medicine

\section{Publish your work in this journal}

The International Journal of General Medicine is an international, peer-reviewed open-access journal that focuses on general and internal medicine, pathogenesis, epidemiology, diagnosis, monitoring and treatment protocols. The journal is characterized by the rapid reporting of reviews, original research and clinical studies across all disease areas.

\section{Dovepress}

A key focus is the elucidation of disease processes and management protocols resulting in improved outcomes for the patient.The manuscript management system is completely online and includes a very quick and fair peer-review system. Visit http://www.dovepress.com/ testimonials.php to read real quotes from published authors. 